\title{
Mordell-Weil Groups of a Hyperkähler Manifold-A Question of F. Campana
}

\author{
Dedicated to Professor Heisuke Hironaka on his 77-th birthday
}

By

Keiji Oguiso*

\begin{abstract}
Among other things, we show that Mordell-Weil groups of finitely many different abelian fibrations of a hyperkähler manifold have essentially no relation, as its birational transformation. Precise definition of the terms "essentially no relation" will be given in Introduction.
\end{abstract}

\section{§1. Introduction-Background and the Statement of Main Result}

Let $M$ be a hyperkähler manifold $M$, i.e., a simply-connected compact Kähler manifold admitting an everywhere non-degenerate global holomorphic 2-form $\sigma_{M}$ s.t. $H^{0}\left(M, \Omega_{M}^{2}\right)=\mathbf{C} \sigma_{M}$. In this note, we assume that $M$ is projective. We denote by $\operatorname{Bir} M$ the group of birational transformations of $M$. A morphism $\varphi: M \longrightarrow B$ onto a normal projective variety $B$ is called an abelian fibration if its generic fiber $M_{\eta}(\ni O)$ is a positive dimensional abelian variety defined over $\mathbf{C}(B)$. The group $M_{\eta}(\mathbf{C}(B))$, called the Mordell-Weil group of $\varphi$ and denoted from now by $\operatorname{MW}(\varphi)$, can be naturally regarded as an abelian subgroup of $\operatorname{Bir} M$. In [Og3] (see also [Og4]), we have shown the following:

Communicated by K. Saito. Received March 12, 2007. Revised September 17, 2007. 2000 Mathematics Subject Classification(s): 14J50, 14J28.

* Department of Economy, Keio University, Hiyoshi Kohoku-ku Yokohama, Japan, and Korea Institute for Advanced Study, 207-43 Cheonryangni-2 dong, Dongdaemun-gu, Seoul 130-722, Korea.

e-mail: oguiso@hc.cc.keio.ac.jp

(C) 2008 Research Institute for Mathematical Sciences, Kyoto University. All rights reserved. 


\section{Theorem 1.1.}

(1) Let $G<\operatorname{Bir} M$. Then either $G>\mathbf{Z} * \mathbf{Z}$ or $G$ is an almost abelian group of rank at most $\operatorname{Max}(1, \rho(M)-2)$, the latter of which is finitely generated.

(2) Assume that $M$ admits 2 different abelian fibrations $\varphi_{i}: M \longrightarrow B_{i}$ s.t. $\operatorname{rank} \operatorname{MW}\left(\varphi_{i}\right)>0(i=1,2)$. Let $f_{i} \in \operatorname{MW}\left(\varphi_{i}\right)$ s.t. ord $f_{i}=\infty$. Then the subgroup $\left\langle f_{1}, f_{2}\right\rangle$ of $\operatorname{Bir} M$ contains $\mathbf{Z} * \mathbf{Z}$ as its subgroup.

Unfortunately, the proof in [Og3], which is based on Tits' alternative [Ti] together with properties of Salem polynomial (cf. [Mc]), tells us no explicit way to find $\mathbf{Z} * \mathbf{Z}$ in $\left\langle f_{1}, f_{2}\right\rangle$. Then $\mathrm{F}$. Campana asked the following:

Question 1.2. Under the same condition as in Theorem (1.1)(2),

$$
\left\langle f_{1}, f_{2}\right\rangle=\left\langle f_{1}\right\rangle *\left\langle f_{2}\right\rangle \simeq \mathbf{Z} * \mathbf{Z}
$$

for suitably chosen $f_{i} \in \operatorname{MW}\left(\varphi_{i}\right)(i=1,2)$ ?

In the view of an observation of Cantat $[\mathrm{Ca}]$, one might ask an even stronger:

Question 1.3. Under the same condition as in Theorem (1.1)(2),

$$
\left\langle f_{1}, f_{2}\right\rangle=\left\langle f_{1}\right\rangle *\left\langle f_{2}\right\rangle \simeq \mathbf{Z} * \mathbf{Z}
$$

for any $f_{i} \in \mathrm{MW}\left(\varphi_{i}\right)$ s.t. $\operatorname{ord}\left(f_{i}\right)=\infty(i=1,2)$ at least when $\operatorname{dim} M=2$ ?

The aim of this note is to give an affirmative answer to the first question in a slightly more general form and a negative answer to the second questions, via an elementary consideration in hyperbolic geometry. An applicability of hyperbolic geometry is suggested by S. Cantat in connection with [Og3].

Definition 1.4. Let $\mathbf{Z}_{+}$be the set of positive integers and let $\Lambda$ be a (possibly infinite) subset of $\mathbf{Z}_{+}$. Let $G$ be a group. We say that subgroups $G_{i}(i \in \Lambda)$ of $G$ have essentially no relation if there are finite index subgroups $H_{i}<G_{i}$ s.t. the group $\left\langle H_{i} \mid i \in \Lambda\right\rangle(\subset G)$ generated by $H_{i}(i \in \Lambda)$ is the free product $*_{i \in \Lambda} H_{i}$.

Our main result is the following:

\section{Theorem 1.5.}

(1) Let $\varphi_{i}: M \longrightarrow B_{i}(i=1,2, \ldots, s)$ be mutually different abelian fibrations on a hyperkähler manifold $M$ s.t. $\operatorname{rank} M W\left(\varphi_{i}\right)=: r_{i}>0$. Then, in 
$\operatorname{Bir} M$, the Mordell-Weil groups $\operatorname{MW}\left(\varphi_{i}\right)(i=1,2, \ldots, s)$ have essentially no relation, i.e., there are finite index subgroups $H_{i}<\operatorname{MW}\left(\varphi_{i}\right)$ s.t.

$$
\left\langle H_{1}, H_{2}, \ldots, H_{s}\right\rangle=H_{1} * H_{2} * \cdots * H_{s} \simeq \mathbf{Z}^{r_{1}} * \mathbf{Z}^{r_{2}} * \cdots * \mathbf{Z}^{r_{s}} .
$$

In particular, for given $g_{i} \in \mathrm{MW}\left(\varphi_{i}\right)$ with ord $g_{i}=\infty$, there are positive integers $m_{i}(i=1,2, \ldots, s)$ s.t.

$\left\langle g_{1}^{m_{1}}, g_{2}^{m_{2}}, \ldots, g_{s}^{m_{s}}\right\rangle=\left\langle g_{1}^{m_{1}}\right\rangle *\left\langle g_{2}^{m_{2}}\right\rangle * \cdots *\left\langle g_{s}^{m_{s}}\right\rangle \simeq \mathbf{Z} * \mathbf{Z} * \cdots * \mathbf{Z}$ (s-factors) .

(2) Let $\varphi_{i}: M \longrightarrow B_{i}\left(i \in \mathbf{Z}_{+}\right)$be mutually different abelian fibrations on a hyperkähler manifold $M$ s.t. $\operatorname{rank} M W\left(\varphi_{i}\right)=: r_{i}>0$. Then, there is an infinite subset $\Lambda$ of $\mathbf{Z}_{+}$s.t. the Mordell-Weil groups $\operatorname{MW}\left(\varphi_{i}\right)(i \in \Lambda)$ have essentially no relation in $\operatorname{Bir} M$, i.e., there are finite index subgroups $H_{i}<\mathrm{MW}\left(\varphi_{i}\right)$ s.t.

$$
\left\langle H_{i} \mid i \in \Lambda\right\rangle=*_{i \in \Lambda} H_{i} \simeq *_{i \in \Lambda} \mathbf{Z}^{r_{i}} .
$$

(3) There are a K3 surface $S$ admitting 2 different abelian (i.e. Jacobian) fibrations $\varphi_{i}: S \longrightarrow \mathbf{P}^{1}$ and elements $f_{i} \in \operatorname{MW}\left(\varphi_{i}\right)$ with ord $f_{i}=\infty(i=1,2)$ s.t. in Aut $S$,

$$
\left\langle f_{1}, f_{2}\right\rangle \simeq \mathbf{Z} / 2 * \mathbf{Z} / 3, \text { whence, }\left\langle f_{1}, f_{2}\right\rangle \neq\left\langle f_{1}\right\rangle *\left\langle f_{2}\right\rangle(\simeq \mathbf{Z} * \mathbf{Z}),
$$

but, for each integer $n \geq 2$,

$$
\left\langle f_{1}^{n}, f_{2}^{n}\right\rangle=\left\langle f_{1}^{n}\right\rangle *\left\langle f_{2}^{n}\right\rangle \simeq \mathbf{Z} * \mathbf{Z} .
$$

As we shall show in Example (4.1), there are K3 surfaces and hyperkähler manifolds admitting infinitely many different abelian fibrations of positive Mordell-Weil rank.

\section{§2. From Hyperkähler Manifold to Hyperbolic Geometry}

In this section, we recall some important theorems which make a clear bridge between groups of birational autmorphisms of a projective hyperkähler manifold and elementary hyperbolic geometry.

Throughout this section, $M$ is a (not necessarily projective) hyperkähler manifold. By $[\mathrm{Be}]$ (see also [GHJ, Part III]), there is a natural symmetric bilinear form called Beauville form, $(*, * *): H^{2}(M, \mathbf{Z}) \times H^{2}(M, \mathbf{Z}) \longrightarrow \mathbf{Z}$. Let $N S(M)$ be the Néron-Severi group of $M$, i.e., the subgroup of $H^{2}(M, \mathbf{Z})$ generated by the first Chern classes of holomorphic line bundles. We regard $N S(M)$ as a (possibly degenerate) sublattice of $H^{2}(M, \mathbf{Z})$ by $(*, * *)$. We denote by $\rho(M)$ the Picard number of $M$, i.e., the rank of $N S(M)$.

The following theorem due to Huybrechts [Hu] (see also [GHJ, Part III] for (1) and [Og2] for (3)) is quite essential: 


\section{Theorem 2.1.}

(1) $M$ is projective iff $N S(M)$ is hyperbolic, i.e., non-degenerate, of signature $(1, \rho(M)-1)$.

(2) Let $g$ be a bimeromorphic automorphism of $M$. Then the natural action $g^{*}$ on $H^{2}(M, \mathbf{Z})$ is a Hodge isometry of $M$. Moreover, the induced representaion $\operatorname{Bir} M \longrightarrow O\left(H^{2}(M, \mathbf{Z})\right)$ has finite kernel.

(3) When $M$ is projective, the induced representaion $\operatorname{Bir} M \longrightarrow O(N S(M))$ has finite kernel.

The statements (1) and (3) make first bridge between Bir $M$ and geometry of the hyperbolic lattice $N S(M)$, when $M$ is projective.

Let $M$ be a projective hyperkähler manifold admitting an abelian fibration $\varphi: M \longrightarrow B$ (See Introduction for the precise definition). The morphism $\varphi$ is given by the complete linear system $|H|$ of some nef divisor $H$ on $M$. This $H$ is of the form $\varphi^{*} H_{B}$, where $h$ is a very ample divisor on $B$. For a general manifold, there are lot of choices of such $H$ (even up to multiple). However, for a hyperkähler manifold, a result due to Matsushita [Ma] says:

Theorem 2.2. $\quad$ The base space $B$ is $\mathbf{Q}$-factorial and of Picard number 1 (and of dimension $\operatorname{dim} M / 2$ ). In particular, the choice of $H$ is unique up to multiple. Moreover, $\left(H^{2}\right)=0$.

As an immediate consequence of Theorems (2.1), (2.2) with Theorem (1.1)(1), we obtain the following bridge between the Mordell-Weil group $\operatorname{MW}(\varphi)$ and geometry of the hyperbolic lattice $N S(M)$ :

Corollary 2.3. Under the notations above, let $O_{H}(N S(M))(\subset$ $O(N S(M)))$ be the stabilizer subgroup of the class $H \in N S(M)$. Then, we have a natural representaion $\mathrm{MW}(\varphi) \longrightarrow O_{H}(N S(M))$ with finite kernel.

\section{§3. From Group Theory and Elementary Hyperbolic Geometry}

(3.1) The next Theorem and its proof are taken from Tits' article [Ti]. This simple theorem is very useful in our proof of Theorems (1.5) and (3.2).

Theorem 3.1. $\quad$ Let $\Lambda$ be a (possibly infinite) subset of $\mathbf{Z}_{+}$of cardinality $|\Lambda| \geq 2$. Let $G$ be a group which acts faithfully on a non-empty set $S$. Let $G_{i}$ $(i \in \Lambda)$ be subgroups of $G$, let $S_{i}(i \in \Lambda)$ be subsets of $S$ s.t. $S \backslash \cup_{i \in \Lambda} S_{i} \neq \emptyset$, and let $p$ be an element of $S \backslash \cup_{i \in \Lambda} S_{i}$. Put $G_{i}^{0}:=G_{i} \backslash\{i d\}$ and $\tilde{S}_{i}:=S_{i} \cup\{p\}$. Suppose that $G_{i}^{0}\left(\tilde{S}_{j}\right) \subset S_{i}$ for all pairs $i \neq j$. Then $\left\langle G_{i} \mid i \in \Lambda\right\rangle=*_{i \Lambda} G_{i}$. 
Proof. Consider $g_{1} g_{2} \cdots g_{m}$, where $g_{k} \in G_{i_{k}}^{0}$ and $i_{1} \neq i_{2} \neq \cdots \neq i_{m}$. Using $|\Lambda| \geq 2$, one can choose an integer $i \in \Lambda$ s.t. $i \neq i_{m}$. Then $G_{i_{m}}^{0}\left(\tilde{S}_{i}\right) \subset S_{i_{m}}$, $G_{i_{m-1}}^{0} G_{i_{m}}^{0}\left(\tilde{S}_{i}\right) \subset S_{i_{m-1}}$, and finally $G_{i_{1}}^{0} G_{i_{2}}^{0} \cdots G_{i_{m-1}}^{0} G_{i_{m}}^{0}\left(\tilde{S}_{i}\right) \subset S_{i_{1}}$. Thus $g_{1} g_{2} \cdots g_{m}(p) \subset S_{i_{1}}$. Hence $g_{1} g_{2} \cdots g_{m}(p) \neq p$ by $p \notin S_{i_{1}}$, and therefore $g_{1} g_{2} \cdots g_{m} \neq i d$.

(3.2) By a hyperbolic lattice $N=(N,(*, * *))$ of rank $\rho$, we mean a pair of a free $\mathbf{Z}$-module $N$ of finite rank $\rho$ and a $\mathbf{Z}$-valued non-degenerate symmetric bilinear form $(*, * *)$ on $N$ with signature $(1, \rho-1)$. We denote by $O(N)$ the group of isometries of $N$. What we need from elementary hyperbolic geometry is the following:

Theorem 3.2. Let $N=(N,(*, * *))$ be a hyperbolic lattice of rank $\rho$. Let $\tilde{\Lambda}$ be a (possibly infinite) subset of $\mathbf{Z}_{+}$of cardinality $|\tilde{\Lambda}| \geq 2$. Suppose that $v_{i}(i \in \tilde{\Lambda})$ are primitive elements of $N \backslash\{0\}$ s.t. $\left(v_{i}^{2}\right)=0$ and $v_{i} \neq \pm v_{j}$ if $i \neq j$, and that $G_{i} \simeq \mathbf{Z}^{r_{i}}\left(i \in \tilde{\Lambda}, r_{i}>0\right)$ are subgroups of $O(N)$ s.t. $G_{i}\left(v_{i}\right)=v_{i}$. Then:

(1) Assume that $|\tilde{\Lambda}|<\infty$, say, $\tilde{\Lambda}=\{1,2, \ldots, s\}$. Then $G_{i}(i=1,2, \ldots, s)$ have essentially no relation.

(2) Assume that $|\tilde{\Lambda}|=\infty$. Then there is an infinite subset $\Lambda$ of $\tilde{\Lambda}$ s.t. $G_{i}$ $(i \in \Lambda)$ have essentially no relation.

Remark 3.3. The statement similar to (1) should be known for experts. In the rest of this section, we shall give a unform, self-contained proof of (1) and (2).

Proof. We denote the scalar extension $N \otimes K$ of $N$ by a field $K$ by $N_{K}$. Whenever we discuss topology, we regard $N_{\mathbf{R}} \simeq \mathbf{R}^{\rho}$ as the Euclidean space by a suitable Euclidean norm $\|*\|$.

1. Let $\mathcal{C}$ be the positive cone of $N$, that is, one of the two connected components of $\left\{x \in N_{\mathbf{R}} \mid(x, x)>0\right\}$. We denote by $\overline{\mathcal{C}}$ the closure of $\mathcal{C}$ in $N_{\mathbf{R}}$, and by $\partial \overline{\mathcal{C}}$, the boundary of $\mathcal{C}$. We define $S:=\{x \in \partial \mathcal{C} \mid\|x\|=1\}$. This space $S$ can be identified with the set of the rays $\left\{\mathbf{R}_{+} x \mid x \in \partial \mathcal{C} \backslash\{0\}\right\}$, by the natural bijection $\mathbf{R}_{+} x \leftrightarrow x /\|x\|$, and is also homeomorphic to the sphere $S^{\rho-2}$. We denote $x /\|x\|$ by $\bar{x}$ and call it the unit vector corresponding to $x$.

2. Let $v$ be a primitive element of $N \backslash\{0\}$ s.t. $\left(v^{2}\right)=0$. Following [Th, Problem 2.5.24], we consider the spaces:

$$
\begin{gathered}
\tilde{L}^{\mathbf{Q}}:=\left\{x \in N_{\mathbf{Q}} \mid(x, v)=1\right\}, \tilde{L}:=\left\{x \in N_{\mathbf{R}} \mid(x, v)=1\right\} ; \\
L^{\mathbf{Q}}:=\tilde{L}^{\mathbf{Q}} / \mathbf{Q} v, L:=\tilde{L} / \mathbf{R} v ;
\end{gathered}
$$




$$
P:=\{x \in \partial \mathcal{C} \mid(x, v)=1\} \subset \tilde{L} .
$$

Here, since $v \in N$ and $(v, v)=0$, the mapping $a v: x \mapsto x+a v, a \in \mathbf{Q}$ (resp. $a \in \mathbf{R}$ ) defines a faithful action of $\mathbf{Q} v\left(\right.$ resp. of $\mathbf{R} v$ ) on $\tilde{L}^{\mathbf{Q}}$ (resp. on $\tilde{L}$ ).

Remark 3.4. Though the rational spaces $\tilde{L}^{\mathbf{Q}}$ and $L^{\mathbf{Q}}$ are not explicitly considered in [Th], it is important to consider these spaces for our proof.

By definition, the space $L^{\mathbf{Q}}$ (resp. $\left.L\right)$ is a $(\rho-2)$-dimensional rational (resp. real) affine space. Or more explicitly, by taking a point in $u \in L^{\mathbf{Q}}$ as 0 and by taking a rational basis of the tangent space at $u$, we can identify $L^{\mathbf{Q}}$ (resp. $L$ ) with the vector space $\mathbf{Q}^{\rho-2}$ (resp. $\mathbf{R}^{\rho-2}$ ). Under these identifications, we have also $L=\left(L^{\mathbf{Q}}\right)_{\mathbf{R}}$. By $p: \tilde{L}^{\mathbf{Q}} \longrightarrow L^{\mathbf{Q}}$ and $p: \tilde{L} \longrightarrow L$, we denote the natural quotient maps. We define the map $\pi$ by $\pi: P \longrightarrow S ; x \mapsto x /\|x\|$.

Let $G \simeq \mathbf{Z}^{r}$ be an abelian subgroup of $O(N)$ s.t. $G v=v$. The group $G$ acts on $\tilde{L}^{\mathbf{Q}}, \tilde{L}, L^{\mathbf{Q}}, L, P$ and $S$. Here the action of $G$ on $S$ is defined by $x \mapsto g(x) /\|g(x)\|$. By definiton, the actions of $G$ on these spaces are equivariant under $p$ and $\pi$.

3. In this paragraph, again closely following [Th, Problem 2.5.14], we shall prove three lemmas, which are crucial in our proof of Theorem (3.2). We use the same notations as in the previous paragraphs.

Lemma 3.5. The map $p \mid P: P \longrightarrow L$ is a homeomorphism and the map

$$
\iota:=\pi \circ(p \mid P)^{-1}: L \longrightarrow S
$$

is a homeomorphism onto $S \backslash\{\bar{v}\}$, i.e., $S$ is a one point compactification of the real affine space $L$ through $\iota$. Moreover, the actions of $G$ on $L$ and $S$ are equivariant under $\iota$.

Proof. The last assertion follows from the last statement in the paragraph 2. Let us show the first two assertions. For a given $x \in \tilde{L}$, there is a unique $\alpha$ s.t. $(x+\alpha v, x+\alpha v)=0$, namely $\alpha=-(x, x) / 2$. Since $(x+\alpha v, v)=1>0$, we have $x+\alpha v \in \partial \mathcal{C}$, and therefore $x+\alpha v \in P$. By the uniqueness of $\alpha$, the map $x+\mathbf{R} v \mapsto x+\alpha v$ is well-defined and gives the inverse $q: L \longrightarrow P$ of $p \mid P$.

By $(v, v)=0$, we have $\pi(P) \subset S \backslash\{\bar{v}\}$. Let $x \in \partial \mathcal{C} \backslash\{0\}$. By the Schwartz inequality, we have $(x, v)>0$ unless $x \in \mathbf{R} v$. Thus, for $x \in S \backslash\{\bar{v}\}$, we have $x /(x, v) \in P$ and $\pi(x /(x, v))=x$. Thus, $x \mapsto x /(x, v)$ gives the inverse of $\pi: P \longrightarrow S \backslash\{\bar{v}\}$. Hence $\iota=\pi \circ q$ satisfies the requirement.

Lemma 3.6. There is a finite index subgroup $G^{\prime}$ of $G$ s.t. $G^{\prime}$ acts on the real affine space $L$ as parallel transformations. Moreover, the action of $G^{\prime}$ on $L$ is faithful and discrete. 
Remark 3.7. For both statements, it seems quite essential that the action $G$ is defined over the lattice $N$, or in other words, over $\mathbf{Z}$. Being parallel transformations is false even for a cyclic orthogonal subgroup of $N_{\mathbf{Q}}$ fixing some non-zero rational vector $v$ with $(v, v)=0$, as there is an infinite-order $3 \times 3$ rotation matrix of rational entries. An account of [Ha, Page 135] seems to miss this point.

Proof. Since $v$ is a primitive vector with $(v, v)=0$, one can choose an integral basis of $v_{N}^{\perp}$ as $\left\langle v, w_{1}, \ldots, w_{n}\right\rangle$. We also choose $u \in N_{\mathbf{Q}}$ s.t. $(v, u)=1$. Such $u$ exists, as $N$ is non-degenerate. Then $\left\langle v, w_{1}, \ldots, w_{n}, u\right\rangle$ forms $\mathbf{Q}$-basis of $N_{\mathbf{Q}}$. In this notation, $\rho=n+2$.

Let $g \in G$. Since $g(v)=v$ and $g\left(v_{N}^{\perp}\right)=v_{N}^{\perp}$, the matrix representation $M(g)$ of $g$ w.r.t. the basis above is of the form:

$$
M(g):=\left(\begin{array}{ccc}
1 & { }^{t} \mathbf{a}(g) & c(g) \\
\mathbf{o} & A(g) & \mathbf{b}(g) \\
0 & { }^{t} \mathbf{o} & d(g)
\end{array}\right) .
$$

Here $A(g)$ is the matrix representation of the action of $g$ on the lattice $N:=$ $v_{N S(M)}^{\perp} / \mathbf{Z} v$ w.r.t. its integral basis $\left\langle\left[w_{i}\right]\right\rangle_{i=1}^{n}$. Thus $A(g)$ is an integral matrix, while $\mathbf{b}(g) \in \mathbf{Q}^{n}$ is, in general, a rational vector. Since $N$ is of negative definite, its orthogonal transformation $A(g)$ is diagonalizable, and the eigenvalues of $A(g)$ are of absolute value 1 . On the other hand, since $A(g)$ is an integral matrix, its eigenvalues are algebraic integers. Thus, by Kronecker's theorem, the eigenvalues of $A(g)$ are roots of 1 . Since $A(g)$ is diagonalizable, $A(g)$ is then of finite order. Let us denote by $m(g)$ the order of $A(g)$.

Let $\left\langle g_{i}\right\rangle_{i=1}^{r}$ be a generator of the free abelian group $G$. Put $g_{i}^{\prime}:=g_{i}^{2 m\left(\tilde{g}_{i}\right)}$ and define the subgroup $G^{\prime}$ of $G$ by $G^{\prime}:=\left\langle g_{1}^{\prime}, g_{2}^{\prime}, \ldots, g_{r}^{\prime}\right\rangle$. Since $G$ is a finitely generated abelian group, $G^{\prime}$ is a finite index subgroup of $G$. Moreover, for each $g \in G^{\prime}$, we have $A(g)=I_{n}$ (the identity matrix) and $d(g)=1$, i.e.,

$$
M(g):=\left(\begin{array}{ccc}
1 & { }^{t} \mathbf{a}(g) & c(g) \\
\mathbf{o} & I_{n} & \mathbf{b}(g) \\
0 & { }^{t} \mathbf{o} & 1
\end{array}\right) .
$$

For $d(g)=1$, observe that $\operatorname{det} M\left(\tilde{g}_{i}\right)= \pm 1$, whence $\operatorname{det} M\left(\tilde{g}_{i}^{2 m\left(\tilde{g}_{i}\right)}\right)=1$, and therefore $\operatorname{det} M(g)=1$ for $g \in G^{\prime}$.

Let $Q \in L$. Then $Q$ is uniquely expressed in the form:

$$
Q=[u]+\sum_{i=1}^{n} x_{i}(Q)\left[w_{i}\right]
$$


and the vector valued function $\mathbf{x}:=\left(x_{i}\right)_{i=1}^{n}$ gives an affine coordinate of both $L$ and $L^{\mathbf{Q}}$. Under this coordinate, one can identify $L^{\mathbf{Q}}=\mathbf{Q}^{n}, L=\left(\mathbf{Q}^{n}\right) \otimes \mathbf{R}=$ $\mathbf{R}^{n}$. Then, by the shape of $M(g)$, the action of $g \in G^{\prime}$ on $L=\mathbf{R}^{n}$ is the parallel transformation by $\mathbf{b}(g) \in \mathbf{Q}^{n}$. This shows the first statement. For the discreteness and faithfulness of the action of $G^{\prime}$ on $L=\mathbf{R}^{n}$, it suffices to show the claim below. Indeed, $\left\langle r\left(g_{i}^{\prime}\right)\right\rangle_{i=1}^{r}$ then forms a part of real basis of $L=\left(\mathbf{Q}^{n}\right) \otimes \mathbf{R}$.

Claim 3.8. The map $r: G^{\prime} \simeq \mathbf{Z}^{r} \longrightarrow \mathbf{Q}^{n} ; g \mapsto \mathbf{b}(g)$ is an injective group homomorphism.

Remark 3.9. Note that the map $(a, b) \mapsto a+b \sqrt{2}$ defines an injective group homomorphism from $\mathbf{Z}^{2}$ to the group $\mathbf{R}$ of the parallel transformations of $\mathbf{R}$, but the image $\{a+b \sqrt{2} \mid a, b \in \mathbf{Z}\}$ is not discrete in $\mathbf{R}$. So, the fact that $G$ acts on the underlying rational space $L^{\mathbf{Q}}$ seems crucial for the discreteness.

Proof. Assume that $\mathbf{b}(g)=\mathbf{o}$. Then, by the shape of $M(g)$, we have $g(v)=v, g\left(w_{i}\right)=w_{i}+a_{i}(g), g(u)=u+c(g) v$. From

$$
(u, u)=(g(u), g(u))=(u, u)+2 c(g)(u, v)=(u, u)+2 c(g),
$$

we have $c(g)=1$. Then, from

$$
\left(u, w_{i}\right)=\left(g(u), g\left(w_{i}\right)\right)=\left(u, w_{i}\right)+a_{i}(g)(u, v)=\left(u, w_{i}\right)+a_{i}(g),
$$

we have $a_{i}(g)=0$. Hence $M(g)=I_{n+2}$, i.e., $g=i d$.

Lemma 3.10. Let $U \subset S$ be a compact neighborhood of the unit vector $\bar{v}$ and let $V$ be a non-empty compact subset of $S \backslash\{\bar{v}\}$. Then, there is a finite index subgroup $H$ of $G$ s.t. $(H \backslash\{i d\})(V) \subset U$.

Proof. Identifying $L$ with $S \backslash\{\bar{v}\}$ by $\iota$ (see Lemma (3.5)), we may assume that

$$
V \subset L \text { and } B:=\{x \in L \mid\|x\|>r\} \subset U \backslash\{\bar{v}\} \subset L
$$

for some $r>0$. Here $\|*\|$ is some Euclidean norm of $L$ (w.r.t. some fixed origin).

Let $G^{\prime} \simeq \mathbf{Z}^{r}$ be a finite index subgroup of $G$ in Lemma (3.6). Then, $G^{\prime}$ acts on $L$ by parallel transformations, say by $\left\{t(g) \in \mathbf{R}^{n} \mid g \in G\right\}$. Since the action of $G^{\prime}$ is discrete by Lemma (3.6), we have $\inf \left\{\|t(g)\| ; g \in G^{\prime} \backslash\{i d\}\right\}>0$.

Thus, there is some positive integer $c$ s.t. $g^{c}(V) \subset B$ for all $g \in G^{\prime} \backslash\{i d\}$. Set $H:=\left\{g^{c} \mid g \in G^{\prime}\right\}$. Since $G^{\prime}$ is a finitely generated abelian group, this set $H$ is a finite index subgroup of $G^{\prime}$. This $H$ satisfies the requirement. 
4. Now we shall complete the proof of Theorem (3.2).

Let us first assume that $|\tilde{\Lambda}|=\infty$. Then the sequence $\left\{\bar{v}_{i}\right\}_{i \in \tilde{\Lambda}}(\subset S)$ has an infinite subsequence which converges to some point $\bar{v}_{\infty} \in S$. Getting rid of the terms $\bar{v}_{i}=\bar{v}_{\infty}$ from this subsequence, we obtain a subsequence $\left\{\bar{v}_{i}\right\}_{i \in \Lambda}$ s.t. $\lim _{i \longrightarrow \infty} \bar{v}_{i}=\bar{v}_{\infty}$ and $\bar{v}_{i} \neq \bar{v}_{\infty}$ for all $i \in \Lambda$. Then $\min _{j \in(\Lambda \backslash\{i\}) \cup\{\infty\}}\left\|\bar{v}_{i}-\bar{v}_{j}\right\|>$ 0 for each $i \in \Lambda$. Thus, we can choose open neighborhoods $V_{i}(\subset S)$ of $\bar{v}_{i}(i \in \Lambda)$ s.t. the closure $\bar{V}_{i}(i \in \Lambda)$ are compact, $\bar{v}_{\infty} \notin \bar{V}_{i}$ for each $i \in \Lambda$ and $\bar{V}_{i} \cap \bar{V}_{j}=\emptyset$ whenever $i \neq j$. Then take open neighborhoods $U_{i}^{0}$ of $\bar{v}_{i}$ s.t. $U_{i}:=\overline{U_{i}^{0}} \subset V_{i}$. We put $\tilde{U}_{i}=U_{i} \cup\left\{\bar{v}_{\infty}\right\}$ for each $i \in \Lambda$. Then, using compactness of $S$ and Lemma (3.10) applied for $\bar{v}=\bar{v}_{i}(i \in \Lambda)$ inductively, we find finite index subgroups $H_{i}$ of $G_{i}(i \in \Lambda)$ s.t. $\left(H_{i} \backslash\{i d\}\right)\left(\cup_{j \neq i} \tilde{U}_{j}\right) \subset U_{i}$. In particular, $\left(H_{i} \backslash\{i d\}\right)\left(\tilde{U}_{j}\right) \subset U_{i}$ whenever $i \neq j$.

Next consider the case where $|\tilde{\Lambda}|<\infty$. In this case, we put $\Lambda=\tilde{\Lambda}$. Since $\Lambda$ is a finite set, we can choose a point $p \in S$ and compact neighborhoods $U_{i}(\subset S)$ of $\bar{v}_{i}(i \in \Lambda)$ s.t. $p \notin U_{i}$ for each $i \in \Lambda$ and $U_{i} \cap U_{j}=\emptyset$ whenever $i \neq j$. We also put $\tilde{U}_{i}=U_{i} \cup\{p\}$ for each $i \in \Lambda$. Then, there are finite index subgroups $H_{i}$ of $G_{i}(i \in \Lambda)$ s.t. $\left(H_{i} \backslash\{i d\}\right)\left(\tilde{U}_{j}\right) \subset U_{i}$ whenever $i \neq j$.

In both cases $\left\langle H_{i} \mid i \in \Lambda\right\rangle=*_{i \in \Lambda} H_{i}$ by Theorem (2.1). This completes the proof.

\section{$\S 4$. Synthesis}

In this section, we shall show Theorem (1.5).

Proof of Theorem (1.5)(1), (2).

Let $v_{i} \in N S(M)$ be the primitive nef element which is proportional to the divisor $H_{i}$ defining $\varphi_{i}$. Then $\left(v_{i}^{2}\right)=0$ by Theorem (2.2). Let $\bar{v}_{i} \in S$ be the unit element corresponding to $v_{i}$. We note that $\bar{v}_{i} \neq \bar{v}_{j}$ if $i \neq j$.

Let $G_{i}:=\operatorname{Im}\left(\mathrm{MW}\left(\varphi_{i}\right) \longrightarrow O(N S(M))\right)$. Then $G_{i}$ preserves $\bar{v}_{i}$ and the kernel of $\mathrm{MW}\left(\varphi_{i}\right) \longrightarrow G_{i}$ is finite by Corollary (1.3). Hence, the result follows from Theorem (3.2).

Proof of Theorem (1.5)(2).

Let $E$ be an elliptic curve. Let us consider the product abelian surface $A:=E \times E$, and its associated Kummer K3 surface $S:=\operatorname{Km} A$. By definition, $S$ is the minimal resolution of the quotient surface $A / \iota$ ( $\iota$ is defined below).

Let $\tilde{p}_{i}: A \longrightarrow E$ be the projection to the $i$-th factor, and let $p_{i}: S \longrightarrow \mathbf{P}^{1}$ be the Jacobian fibration on $S$, induced by $\tilde{p}_{i}$.

Let

$$
\tilde{f}_{2}:=\left(\begin{array}{ll}
1 & 1 \\
0 & 1
\end{array}\right), \tilde{f}_{1}:=\left(\begin{array}{ll}
1 & 0 \\
1 & 1
\end{array}\right), \iota:=\left(\begin{array}{cc}
-1 & 0 \\
0 & -1
\end{array}\right)
$$


and put $\tilde{G}:=\left\langle\tilde{f}_{1}, \tilde{f}_{2}\right\rangle$ in $\operatorname{SL}(2, \mathbf{Z})$. Then $\tilde{G}=\operatorname{SL}(2, \mathbf{Z})$ and $\operatorname{SL}(2, \mathbf{Z})$ acts faithfully on $A$ by $\tilde{f}_{2}(x, y)=(x+y, y), \tilde{f}_{1}(x, y)=(x, x+y)$. By the shape of $\tilde{f}_{i}$, we see that $\tilde{f}_{i} \in \mathrm{MW}\left(\tilde{p}_{i}\right)$ and ord $\tilde{f}_{i}=\infty$. Since $\tilde{f}_{i} \iota=\iota \tilde{f}_{i}$, we also see that $\tilde{f}_{i}$ descends to $f_{i} \in \mathrm{MW}\left(p_{i}\right)$ and ord $f_{i}=\infty$.

Set $G:=\left\langle f_{1}, f_{2}\right\rangle$ in Aut $S$. Then, $G \simeq \operatorname{SL}(2, \mathbf{Z}) /\langle\iota\rangle=\operatorname{PSL}(2, \mathbf{Z})$.

We shall show that $\left(S, f_{1}, f_{2}\right)$ satisfies the requirement.

It is well-known that $\operatorname{PSL}(2, \mathbf{Z}) \simeq \mathbf{Z} / 2 * \mathbf{Z} / 3$ (see eg. [Kn, Page 147]). In particular, $\left\langle f_{1}, f_{2}\right\rangle \neq\left\langle f_{1}\right\rangle *\left\langle f_{2}\right\rangle$; if otherwise, $\mathbf{Z} / 2 * \mathbf{Z} / 3 \simeq \mathbf{Z} * \mathbf{Z}$, a contradiction.

It remains to show that $\left\langle f_{1}^{n}, f_{2}^{n}\right\rangle=\left\langle f_{1}^{n}\right\rangle *\left\langle f_{2}^{n}\right\rangle$ for each $n \geq 2$. Our proof of this fact closely follows [Ha, Example 1]. Note that $\operatorname{PSL}(2, \mathbf{Z})<\operatorname{PGL}(2, \mathbf{Z})$.

In $\operatorname{PGL}(2, \mathbf{Z})$, we put

$$
g_{2}:=f_{2}^{n}=\left(\begin{array}{ll}
1 & n \\
0 & 1
\end{array}\right), g_{1}:=f_{1}^{n}:=\left(\begin{array}{ll}
1 & 0 \\
n & 1
\end{array}\right), j:=\left(\begin{array}{ll}
0 & 1 \\
1 & 0
\end{array}\right) .
$$

Then $j^{2}=i d$ and $g_{1}=j g_{2} j$ in $\operatorname{PGL}(2, \mathbf{Z})$. So, if there would be a non-trivial relation among $g_{1}$ and $g_{2}$, say, $h\left(g_{1}, g_{2}\right)=i d$, then substituting $g_{1}^{l}=j g_{2}^{l} j$ into the relation $h\left(g_{1}, g_{2}\right)=i d$, we would have a non-trivial relation among $g_{2}$ and $j$. Thus, we suffice to show that $\left\langle g_{2}, j\right\rangle=\left\langle g_{2}\right\rangle *\langle j\rangle$ in $\operatorname{PGL}(2, \mathbf{Z})$.

To prove this claim, we consider the natural fractional linear action of $\operatorname{PGL}(2, \mathbf{Z})$ on $\mathbf{P}^{1}=\mathbf{C} \cup\{\infty\}$, and the following subsets and a point of $\mathbf{P}^{1}$ :

$U_{1}:=\{z \in \mathbf{C}|| z \mid<1\}, U_{2}:=\{z \in \mathbf{C}|| \operatorname{Re} z \mid>1\} \cup\{\infty\}, P:=2 \sqrt{-1} \notin U_{1} \cup U_{2}$.

Then $j\left(U_{2} \cup\{P\}\right) \subset U_{1}$ and $g_{2}^{k}\left(U_{1} \cup\{P\}\right) \subset U_{2}$ for each $k \neq 0$ (by $n \geq 2$ ). Thus, by Theorem (2.1), we have $\left\langle g_{2}, j\right\rangle=\left\langle g_{2}\right\rangle *\langle j\rangle$, and we are done.

Example 4.1. Let $\varphi: S \longrightarrow \mathbf{P}^{1}$ be a (necessarily projective) Jacobian K3 surface s.t. $\operatorname{rank} \operatorname{MW}(\varphi)=18$ and $\rho(S)=20$, which are maximum. Such a Jacobian $\mathrm{K} 3$ surface exists by $[\mathrm{Co}]$ and $[\mathrm{Ni}]$. It is also known that, given a Jacobian K3 surface $S^{\prime}$, one can find such a Jacobian K3 surface $S$ arbitrarily close to $S^{\prime}$ in the period domain ([Og1]). In Aut $S$ and Aut $S^{[n]}$, we can find an infinite sequence of subgroups

$$
\mathbf{Z}^{18} \subset \mathbf{Z}^{18} * \mathbf{Z}^{18} \subset \mathbf{Z}^{18} * \mathbf{Z}^{18} * \mathbf{Z}^{18} \cdots \subset *_{s} \mathbf{Z}^{18} \subset \cdots .
$$

Here $S^{[n]}$ is the Hilbert scheme of $n$-points on $S$, which is a hyperkähler manifold of dimension $2 n$ ([Be]).

Proof. Let $e \in \operatorname{Pic} S \simeq N S(S)$ be the fiber class of $\varphi$. Since $\varphi$ has at least three singular fibers (see eg., [Ca], [VZ]), the subgroup Aut $\varphi:=\{f \in$ 
Aut $S \mid f^{*}(e)=e$ \} of Aut $S$ is an almost abelian group (of rank 18). On the other hand, since $\rho(S)=20$, we have $\mathbf{Z} * \mathbf{Z} \subset$ Aut $S$ by [Og3]. In particular, Aut $S$ is not an almost abelian group.

Hence the coset Aut $S /$ Aut $\varphi$ is an infinite set. Thus, the Aut $S$-orbit of $e$ is an infinite set as well. Therefore, $S$ admits infinitely many different Jacobian fibrations with Mordell-Weil rank 18. Now the result for $S$ follows from Theorem (1.5)(2). The Mordell-Weil group of a Jacobian fibration $S \longrightarrow$ $\mathbf{P}^{1}$ can be naturally embedded into the Mordell-Weil group of the induced abelian fibration $S^{[n]} \longrightarrow \mathbf{P}^{n}$. This implies the result for $S^{[n]}$.

\section{Acknowledgements}

I would like to express my thank to Professors F. Campana, S. Cantat, J.M. Hwang, M. Paun, and J. Winkelman for their valuable discussions, mostly in Johns Hopkins University, Luminy and Oberwolfach. Finally, I would like to express my deep thank to the referee for valuable comments, especially the comment which indicates a way to divide an algebro-geometric part and a purely elementary hyperbolic geometrical part cleanly. This makes our explanation much cleaner.

\section{References}

[Be] A. Beauville, Variétés Kähleriennes dont la première classe de Chern est nulle, J. Differential Geom. 18 (1983), no. 4, 755-782.

[Ca] S. Cantat, Sur la dynamique du groupe d'automorphismes des surfaces $K 3$, Transform. Groups 6 (2001), no. 3, 201-214.

[Co] D. A. Cox, Mordell-Weil groups of elliptic curves over $\mathbf{C}(t)$ with $p_{g}=0$ or 1 , Duke Math. J. 49 (1982), no. 3, 677-689.

[GHJ] M. Gross, D. Huybrechts and D. Joyce, Calabi-Yau manifolds and related geometries, Springer, Berlin, 2003.

[Ha] P. de la Harpe, Free groups in linear groups, Enseign. Math. (2) 29 (1983), no. 1-2, 129-144.

[Hu] D. Huybrechts, Compact hyper-Kähler manifolds: basic results, Invent. Math. 135 (1999), no. 1, 63-113. Erratum: "Compact hyper-Kähler manifolds: basic results" Invent. Math. 152 (2003), no. 1, 209-212.

[Kn] T. Kondo, Group Theory. I-III (Japanese) Second edition, Iwanami Shoten, Tokyo, 1991.

[Ma] D. Matsushita, On fibre space structures of a projective irreducible symplectic manifold, Topology 38 (1999), 79-83, Addendum: On fibre space structures of a projective irreducible symplectic manifold, Topology 40 (2001), 431-432.

[Mc] C. T. McMullen, Dynamics on $K 3$ surfaces: Salem numbers and Siegel disks, J. Reine Angew. Math. 545 (2002), 201-233.

[Ny] K. Nishiyama, Examples of Jacobian fibrations on some $K 3$ surfaces whose MordellWeil lattices have the maximal rank 18, Comment. Math. Univ. St. Paul. 44 (1995), no. 2, 219-223 
[Og1] K. Oguiso, Local families of $K 3$ surfaces and applications, J. Algebraic Geom. 12 (2003), no. 3, 405-433.

[Og2] Bimeromorphic automorphism groups of non-projective hyperkähler manifolds - a note inspired by C. T. McMullen, J. Differential Geom. 78 (2008), 163-191.

[Og3] _ Tits alternative in hypekähler manifolds, Math. Res. Lett. 13 (2006), no. 2$3,307-316$.

[Og4] Salem polynomials and birational transformation groups for hyperKähler manifolds, Sūgaku 59 (2007), no. 1, 1-23.

[Th] W. P. Thurston, Three-dimensional geometry and topology. Vol. 1, Princeton Univ. Press, Princeton, NJ, 1997.

[Ti] J. Tits, Free subgroups in linear groups, J. Algebra 20 (1972), 250-270.

[VZ] E. Viehweg and K. Zuo, On the isotriviality of families of projective manifolds over curves, J. Algebraic Geom. 10 (2001), no. 4, 781-799. 